\title{
PERFORMANCE ANALYSIS OF SUCCESSIVE INTERFERENCE CANCELLATION WITH MODIFIED QUADRATIC CONGRUENCE AND HADAMARD CODES FOR OPTICAL CDMA
}

\author{
Tawfig Eltaif $^{1 \mathrm{a}}$, Hossam M. H. Shalaby ${ }^{2}$, Sahbudin Shaari ${ }^{1}$, Mohammad M. N. Hamarsheh ${ }^{3}$ \\ ${ }^{1}$ Photonics Technology Laboratory (PTL), Institute of Micro Engineering and Nanoelectronics, \\ Universiti Kebangsaan Malaysia, 43600 UKM Bangi, Selangor, MALAYSIA \\ 2Elect. Eng. Dept, Fac. of Engineering, Alexandria University, Alexandria 21544, EGYPT. \\ ${ }^{3}$ Dept. of Telecommunication Engineering (TCE), Fac. of Information Technology, Arab American University, Jenin, \\ P.O. BOX $1: 240$ Jenin, P.O. Box $2: 13$ Zababdeh, Palestine \\ atefosat@vlsi.eng.ukm.my
}

\begin{abstract}
One of the techniques used to mitigate the problem of cross correlation is successive interference cancellation (SIC). This technique has been inspired of multi user detection technique (MUD), and can increase the capacity of optical CDMA system. A detailed analysis of this system is theoretically analyzed, taking into account multiple-access interference (MAI). In addition, the system performance is analyzed with both modified quadratic congruence (MQC) code and Hadamard codes. We have also compared the performance of this system (in terms of bit error rate) with conventional scheme. It has been shown that our proposed scheme performs better than the conventional scheme, and it has been found that signature code sets with higher weight are preferred in our SIC scheme.
\end{abstract}

Keywords: Optical code-division multiple access (OCDMA), multiple-access interference (MAI), successive interference cancellation (SIC), Hadamard code, Modified quadratic congruence code (MQC).

\section{Introduction}

Up to this time, optical CDMA methods have been studied and developed for optical systems. The main methods are used optical orthogonal code (OOC) $[1,2]$, which can be generated using one of the following devices: optical delay lines or optical switches [1,2]; fiber Bragg grating (FBG) or arrayed waveguide gratings (AWGs) [3]. OCDMA using OOCs has many problems, such as a limitation on the number of distinct code sequences and low optical power because there are fewer $1 \mathrm{~s}$ than $0 \mathrm{~s}$.

One of the key issues for OCDMA is to reduce the multiple access interference (MAI) among the users, and to come across this type of the problems many techniques have been studied on interference cancellation, such as Salehi and Brackett [4] have used an optical hardlimiter.

The authors would like to thank the Malaysian Ministry of Science, Technology and Environment for sponsoring this work under project UKMGUP-BTT-07-26-177.
Double optical hardlimiters placed before and after the optical correlator have been proposed in [5]. Lin and $\mathrm{Wu}$ in [6] have proposed a synchronous OCDMA system with an adaptive optical hardlimiter placed after the correlator receiver, they show the performance can be improved, compared with system with double hardlimiter. Recently one technique inspired by radio frequency communications, which is called multi-user detection (MUD) technique which typically employed in optical CDMA [7], to improve the capacity and overall throughout of the system, and it is known that optimum multi-user detection (MUD) has a much better theoretical performance than conventional detection [8]. Inspired of MUD we have been introducing the successive interference cancellation (SIC) [9]. In [10, 11] we have analyzed SIC scheme using optical orthogonal code (OOC) for optical CDMA, where the system is very efficient in elimination the effect of the multiple access interference.

Moreover, we have analyzed this technique in [12] using modified prime sequence code, and it is shown that the bit error rate performance of the system significantly improved at each stage of cancellation process using both OOC code and prime code and the system can suppress the MAI and increase the system capacity.

\section{Basic Principle of SIC Scheme}

The basic idea of this scheme is simple, it detect and demodulates the strongest user signal currently present in the overall received signal. Figure 1 shows the block diagram of a SIC receiver. In the next stage after this user has been detected and demodulated; the original signal is regenerated and subtracted from the overall received signal to get a new received signal [9-12]. In general algorithm the successive cancellations are carried as follows: i) Recognize the strongest signal (one with maximum correlation value), ii) Decode the strongest user, iii) Regenerate the strongest users' signal using its chip sequence, iv) Cancel the 
strongest user, v) Repeat (until all users are decoded or a permissible number of cancellations are achieved).

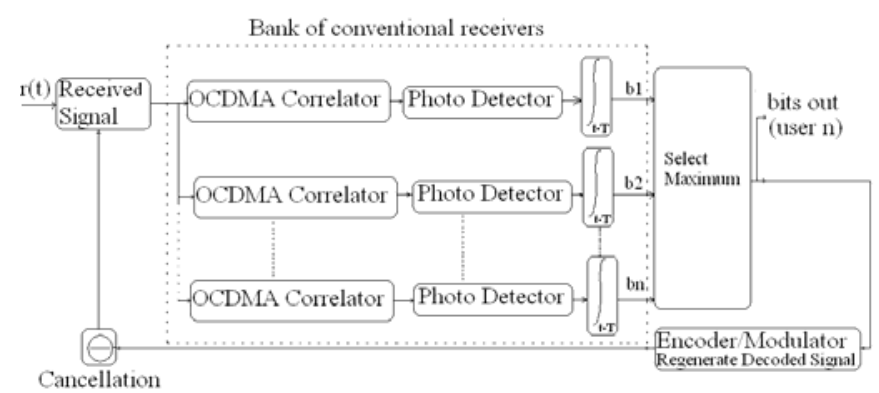

Figure 1. SIC receiver block diagram.

Several signature code sets have been proposed for optical CDMA, such as Hadamard, modified prime code, and modified quadratic congruence (MQC) code [13-18]. Each of these signature code sets can be represented by its length, and weight. The length prime code families have various length sequence code, weight as well. According to $[13,14]$ it is known that an $(N x N)$ Hadamard matrix of 1's and 0 's has the property that any row differs from any other row in exactly $N / 2$ positions, therefore let $c_{n}(i)$ denote the $i$ th element of the $n$th code sequence, the code properties can be expressed as:

$$
\sum_{i=1}^{N} c_{n}(i) c_{l}(i)=\left\{\begin{array}{ll}
\frac{N}{2} ; & n=l \\
\frac{N}{4} ; & n \neq l
\end{array} \quad n, l \in(2,3, \ldots, N) .\right.
$$

In Hadamard code $(N-1)$ code sequence can be constructed each one has weight equal to $N / 2$ and a length $N$.

\section{Performance Analysis}

In this section, we have analyzed and presented the SIC scheme more in details with modified quadratic congruence (MQC) code. More details of MQC can be found in [15], where MQC code shows that is much better than Hadamard codes for SAC optical CDMA systems. Therefore under the worst case i.e., $\tau_{n}=0$, for all n, synchronized condition [1], the cross correlation function $I_{x y}$ between any pair of code sequences $\mathrm{x}$ and $\mathrm{y}$ is given by [15]:

$$
I_{x, y}= \begin{cases}p+1 ; & x=y \\ 0 ; & x \text { and } y \text { are in the same group } \\ 1 ; & x \text { and } y \text { are in different group }\end{cases}
$$

Assuming that each user is assigned a unique prime sequence code of length $p^{2}+p$ with weight $(p+1)$ and considering the total number of users $N=p^{2}$. Then the receiver signal $r(t)$ at the front end of the receiver is modeled as follows:

$$
r(t)=\sum_{n=1}^{N=p^{2}} A_{n} b_{n}\left(t-\tau_{n}\right) \sum_{i=1}^{p^{2}+p} c_{n}^{i}\left(t-\tau_{n}\right)+n(t)
$$

Where; $N$ is the total number of users; $A_{n}$ is the signal strength of the $n^{\text {th }}$ user; $b_{n}(t)$ is the bit sequence of $n^{\text {th }}$ user; $c_{n}(t)$ is the spreading chip sequence of the $n^{\text {th }}$ user; $n(t)$ is the noise signal (thermal noise); and $\tau_{n}$ is the time delay of the $n^{\text {th }}$ user.

Detailed analysis of the SIC scheme for incoherent DSOCDMA system can be found in [9-12]. After $\mathrm{j}^{\text {th }}$ cancellation, the decision variable for the $(j+1)^{\text {th }}$ user taking in account the code properties indicated in Eq. (1), is given by:

$$
Z_{j+1}=\zeta_{j+1}+l_{j+1}
$$

Where

$$
\begin{gathered}
\zeta_{j+1}=\frac{A_{j+1} b_{(j+1)}}{p} \\
l_{j+1}=\frac{1}{p^{2}}\left[\sum_{n=j+2}^{N=p^{2}} A_{n} b_{n} I_{n, j+1}\left(\tau_{n, j+1}\right)-\right. \\
\left.\sum_{i=1}^{j} l_{i} I_{i, i+1}\left(\tau_{i, i+1}\right)\right]+n_{j+1}(t)
\end{gathered}
$$

In the above expression, the first term is MAI of the uncancelled users; and the second term is duo to cumulative noise from imperfect cancellation. The original prime code is analyzed by using a Gaussian approximation. The negative effects of shot noise, effect of the receiver's dark current, and other sources of noise are neglecting in order to focus only on the interference (i.e., MAI), created by other simultaneous users, furthermore the thermal noise.

When $N$ represented the users are transmitting simultaneously, the total interference at a given receiver is 
the superposition of $(N-1)$ different cross correlation functions. If the $(N-1)$ interference is uncorrelated, then the variance of the total interference is equal to the sum of the variance of the $(N-1)$ cross correlation functions. We can define the variance of $l_{j+1}$ conditioned on $A_{n}$ as follows:

$$
\begin{aligned}
& \left.\Gamma^{2}{ }_{j+1}\right|_{-} M A I=\frac{1}{p^{2}}\left[\sum_{n=j+2}^{N=p^{2}} A_{n}{ }^{2} b_{n}^{2} \cdot \operatorname{var}\left[I_{n, j+1}\left(\tau_{n, j+1}\right)\right]+\right. \\
& \left.\sum_{i=1}^{j} \Gamma^{2}{ }_{i} \cdot \operatorname{var}\left[I_{i, i+1}\left(\tau_{i, i+1}\right)\right]\right]
\end{aligned}
$$

And the variance of cross correlation in (2) is found as [11]:

$$
\operatorname{var}\left(I_{n, j+1}\right) \approx \frac{1}{p^{2}+p}
$$

Substituting (8) into (7) we get the variance of the noise in the decision variable:

$$
\left.\Gamma_{j+1}^{2}\right|_{-} M A I=\frac{1}{p^{2}\left(p^{2}+p\right)}\left[\sum_{n=j+2}^{N=p^{2}} A_{n}^{2}+\sum_{i=1}^{j} \Gamma_{i}^{2}\right]
$$

Furthermore the thermal noise has a Gaussian distribution effect has been considered:

$$
\sigma_{t h}=4 K_{b} T_{n} B / R_{L}
$$

Where $B$ is the noise-equivalent electrical bandwidth of the receiver in hertz; $K_{b}$ is the Boltzmann's constant in joules per Kelvin $=1.38 \times 10^{-23} \mathrm{JK}^{-1} ; T_{n}$ is the absolute receiver noise temperature in Kelvin; and $R_{l}$ is the receiver load resistor in ohm.

The responsivity of the PDs is given by $\Re=\eta e / h v_{c}$. Here, $\eta$ is the quantum efficiency, $e$ is the electron's charge, $\mathrm{h}$ is the Plank's constant, and $v_{c}$ is the central frequency of the original broadband optical pulse.

The signal to noise ratio function (SNR) is represented as the ratio of the squared of the peak of the autocorrelation function to the variance of the amplitude of the interference. Then from (4), (9) and (10), we can get the signal to noise ratio function of $A_{n}$

$$
S N R_{j+1}=\frac{\mathfrak{R}^{2} A_{j+1}^{2} / p^{2}}{\frac{\mathfrak{R}^{2}}{p^{2}\left(p^{2}+p\right)}\left[\sum_{n=j+2}^{N=p^{2}} A_{n}^{2}+\sum_{i=1}^{j} \Gamma^{2}{ }_{i}\right]+4 K_{b} T_{n} B / R_{L}}
$$

It is clear from SNR equation the variance of the amplitude of the cross correlation function $\left(\left.\Gamma^{2}{ }_{j+1}\right|_{-} M A I\right)$ (the denominator of the SNR) decreases with prime number $p$. To calculate the bit error rate, we shall assume the noise $l_{j+1}$ is Gaussian with zero mean and variance $\Gamma_{j+1}^{2}$. Then the probability of error, conditioned on the $A_{n}$, is given below as follows:

$$
B E R_{j+1}=Q\left(\frac{\left|\zeta_{j+1}\right|}{\Gamma_{j+1}}\right)=Q\left(\sqrt{S N R_{j+1}}\right)
$$

\section{Numerical Results}

In this section, numerical results are presented to highlight the advantages of this system (SIC). For the analysis, we have used modified quadratic congruence codes as a signature codes when the prime number set has a length 7, 11, and 13, Hadamard code also has been tested for this scheme where the number of users $N=128$. The typical parameters used in our analysis given in table 1 . Figure 2 shows the relation between the number of active users and the SNR using both sequence codes. It is shown that in this figure, using effective power of $-20 \mathrm{dBm}$, the SNR of each curve ends at the point where the number of active users is equal to the code size. For a given code weight, the SNR decreases as the number of active users increases, a degradation in the SNR implies worsening in the error probability. That is the more simultaneous users, the poorer system performance becomes. Furthermore, the SNR is improved as the prime number increased; it is clear from the figure number of active users increase with prime number. Hence, the SNR increased by $5 \mathrm{~dB}$, for constant number of active users (40) for both prime numbers $(p=11,13)$, whereas the SNR increased more than $(12 \mathrm{~dB})$ between prime numbers $(p=7,11)$. Moreover it is shown that the SNR of the system with MQC code is higher than Hadamard code especially when the number of prime code increases. 
Therefore, SIC system can be mitigate MAI, and significantly increase the system capacity.

Figure 3 shows the system's BER versus the number of active users, where the comparison of BER performance between modified quadratic congruence codes and Hadamard codes has been established. Moreover, for different numbers of users, it is shown that the bit error rate improves as the number of prime code increases, and it is clearly shown from the figure MQC codes has much lower BER than the Hadamard code. (i.e., better performance).

Table 1. Typical parameters used for the calculation

\begin{tabular}{ll}
\hline Parameter & Value \\
Operation Wavelength & $193.1 \mathrm{THz}$ \\
PD quantum efficiency & 0.6 \\
Receiver noise temperature & $300 \mathrm{k}$ \\
Receiver load resistor & $1030 \Omega$ \\
Electrical equivalent bandwidth & $80 \mathrm{MHz}$ \\
\hline
\end{tabular}

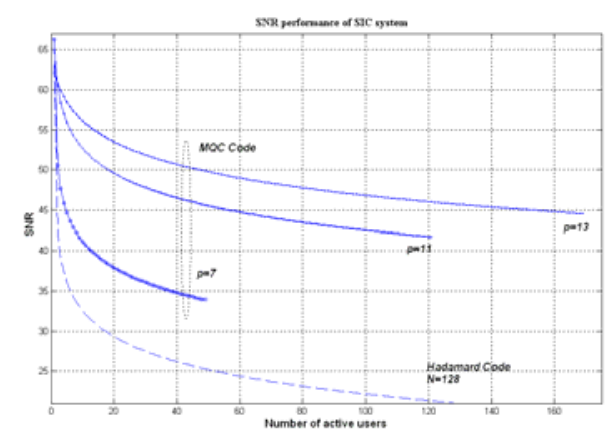

Figure 2. SNR versus number of active users under effective power ($20 \mathrm{dBm})$

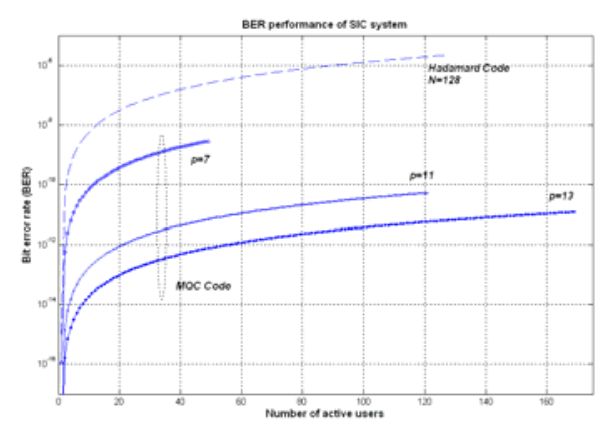

Figure 3. BER versus number of active users under effective power ($20 \mathrm{dBm})$

In figure 4 the BER function for the system with and without cancellation has been plotted for the sake of comparison. The bit error rate from the analysis obtains shows that the cancellation scheme has better performance than conventional scheme. Assuming that prime number set as a length 13 , it is clearly for similar BER performance $\left(10^{-}\right.$ ${ }^{12}$ ), less than 20 users can be active with conventional scheme, which can be increased to 60 users with SIC cancellation scheme, giving substantial increase in capacity.

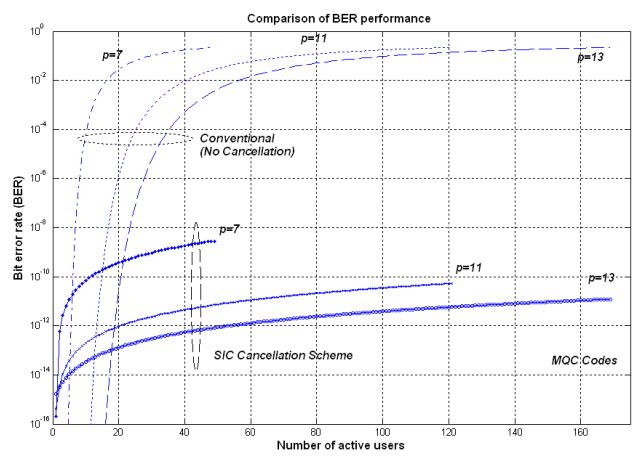

Figure 4. Comparison of BER performance

\section{Conclusion}

In this paper we have presented an analysis of the performance of SIC system using both modified quadratic congruence and Hadamard codes, taking in to account effects of thermal noise sources. The BER performance of both codes for this system have been compared and it is clear that SIC scheme gives better results of BER when the MQC code used, and the BER improves with increasing prime code $p$. Clearly the MQC code gets a good performance results compared to Hadamard code, which means using MQC code for this system can effectively suppress the effect of multiple access interference (MAI). When $P_{e r}$ is large, the Multiple access interference (MAI) acts as the main factor to limiting the system performance. However, the system shows much lower BER performance with SIC cancellation as compared to the one without cancellation. Hence, much more number of users can accommodate by our proposed system. This increase in the number of users results from the cancellation process. Indeed, at each stage of the cancellation, the number of interfering users decreases, the cross correlation between 
users decreases, the SNR increases, and finally the BER decreases.

\section{REFERENCES}

[1] J. A. Salehi, "Code division multiple-access techniques in optical fiber networks-Part I," IEEE Trans. Commun., 37, no. 8, 824842 (1989).

[2] S. Park, K. Tsukamoto, and S. Komaki, "Proposal of Direct Optical Switching CDMA for Cable-To-The-Air System and Its Performance Analysis," IEICE Trans. Comm., 81-B, no. 6, 1188-1196 (1998).

[3] J. Huang, D. Hsu, and Y. Wang, "Photonic CDMA Networking with Spectrally Pseudo-Orthogonal Coded Fiber Bragg Gratings," IEICE Trans. Comm., 83-B, no. 10, 2331-2340 (2000).

[4] J. A. Salehi and C. A. Brackett, "Code division multiple-access techniques in optical fiber networks-Part II: Systems performance analysis,"IEEE Trans. Commun., 37, 834-842 (1989).

[5] T. Ohtsuki, K. Sato, I. Sasase, and S. Mori, "Direct-detection optical synchronous CDMA systems with double optical hard-limiters using modified prime sequence codes," J. Select. Areas Commun., 14, pp. 1897-1887, (1996).

[6] C. L. Lin and J. Wu, "A synchronous fiber-optic CDMA system using adaptive optical hardlimiters," J. Lightwave Technol., 16, 13931403 (1998).

[7] M. B. Pearce and B. Aazhang, "Multiuser Detection for Optical Code Division Multiple Access Systems," IEEE Trans. Commun, 42, no. 2/3/4, 1801-1810 (1994).

[8] S. Verdu, "Adaptive Multiuser Detection," in Proceedings of IEEE on International Symposium on Spread Spectrum Techniques and Applications, Oulu, Finland, 43- 50 (1994).

[9] T Eltaif, H M H Shalaby and S Shaari, "A Novel Successive Interference Cancellation Scheme in OCDMA System", in Proceedings of IEEE Conference on.(ICSE, 2006), 299 - 303 (2006).

[10] T Eltaif, H M H Shalaby, S Shaari, \&M. M. N. Hamarsheh. "A proposal of successive interference cancellation scheme in optical CDMA systems," optic. Eng. SPIE. Vol. 47, no. 3, 035006(1-6) (2008).

[11] T Eltaif, H M H Shalaby, S Shaari, \&M. M. N. Hamarsheh. "Analysis of Successive Interference Cancellation Scheme using OOC code in Optical CDMA Systems," in Proceeding of International Conference on Advanced Communication Technology (IEEE. ICACT, 2008), 2023-2026 (2008).

[12] T Eltaif, H M H Shalaby, S Shaari, \&M. M. N. Hamarsheh. "Performance analysis of successive interference cancellation scheme for optical CDMA systems using modified prime sequence codes," in Proceedings of the SPIE, Volume 6837, 68370Y (2008).
[13] E. D. J. Smith, R. J. Blaikie, and D. P. Taylor, "Performance enhancement of spectral-amplitude-coding optical CDMA using pulse-position modulation," IEEE Trans. Commun., 46, 11761185 (1998).

[14] M. Kavehrad and D. Zaccarin, "Optical code-division-multiplexed systems based on spectral encoding of noncoherent sources," J. Lightwave Technol., 13, no. 3, 534-545 (1995).

[15] W. Zou, H. M. H. Shalaby, and H. G. Shiraz, "Modified quadratic congruence codes for fiber Bragg-grating-based spectralamplitude-coding optical CDMA systems," J. Lightwave Technol., LT-19, 1274-1281 (2001).

[16] P. Sweeney, Error Control Coding: From Theory to Practice, University of Surrey, Guildford, UK. (2002).

[17] W. C. Kwong, P. A. Perrier, and P. R. Prucnal, "Performance comparison of asynchronous and synchronous code-division multiple-access techniques for fiber-optic local area networks," IEEE Transactions on Communications, 39, 1625-1634 (1991).

[18] G.-C. Yang and W. C. Kwong, "Performance analysis of optical CDMA with prime codes," Electronics Letters, 31, 569-570 (1995). 